\title{
DISPOSICIÓN A USAR CONDÓN EN LOCALIDADES CON ALTA MOVILIDAD POBLACIONAL DE MÉXICO Y CENTROAMÉRICA
}

\author{
MARIO SALVADOR SÁNCHEZ-DOMÍNGUEZ \\ RENÉ LEYVA-FLORES \\ MARTA CABALLERO GARCÍA \\ CÉSAR INFANTE XIBILLE*
}

\section{Resumen}

El objetivo del estudio es conocer los factores asociados a la disposición para uso de condón en población general que vive en contextos de alta movilidad poblacional en México y Centroamérica. Se analizó una encuesta realizada en once localidades de México y Centroamérica, empleando un modelo multivariado de regresión logística para identificar las variables asociadas a la disposición a usar condón. La población dispuesta a usar condón se caracteriza por ser hombres (RP), jóvenes, solteros, estudiantes, con escolaridad mayor de seis años, con nivel medio-alto de conocimientos acerca de las ITS/VIH/ SIDA y que se perciben en riesgo para contraer ITS/VIH. Este estudio permite conocer las características de la población que vive en contextos de alta movilidad poblacional, y su relación con la disposición a adoptar una medida preventiva para el VIH/SIDA.

Palabras clave: disposición al uso, condón, movilidad poblacional, SIDA, medidas de prevención.

\footnotetext{
* Todos los autores adscritos al Instituto Nacional de Salud Pública. Correos electrónicos: mario.sanchez@insp.mx / rene.leyva@insp.mx / martacg@uaem.mx / cesar.infante@insp.mx
} 


\section{Abstract}

The aim of the study was to determine factors associated with readiness for condom use in the general population living in conditions of high population mobility in Mexico and Central America. We analyzed a survey conducted in eleven locations in Mexico and Central America, using a logistic regression model to identify variables associated with willingness to use condoms. The people willing to use condoms is characterized by men (RP), youth, singles, students, schooling more than six years, with medium to high level of knowledge about STIS and HIV/AIDS and perceived risk is STI/HIV. This study allows to determine the characteristics of the population living in conditions of high population mobility and its relationship with the willingness to take preventive measures for HIV/AIDS.

Keywords: Use disposition, Condom, population mobility, AIDS, prevention measurements. 


\section{INTRODUCCIÓN}

Desde los primeros casos reportados de VIH/SIDA se ha identificado una asociación de la epidemia con la movilidad poblacional. Sin embargo, se ha reconocido ampliamente que no son los movimientos poblacionales por sí mismos los que determinan el riesgo para la transmisión del viH, sino los contextos y tipos de interacciones sociales que ocurren en los lugares en donde se da la movilidad poblacional (Bronfman, 2003) (Joint United Nations Programme on HIV/AIDS, 1997).

En este sentido, diferentes autores, han documentado la desigualdad social, económica, cultural y legal de los migrantes indocumentados, lo cual también se ha reportado como determinantes de mayor vulnerabilidad social y ante la infección por el viH en comparación con otros grupos sociales (Bronfman, 2003) (Shedlin, 2006) (Shedlin, 2005).

En las localidades fronterizas incluidas en el estudio se presenta una elevada movilidad poblacional de personas sin documentos migratorios; situación que los hace vulnerables a la violación de derechos humanos y civiles, acompañadas en ocasiones de violencia física. En este contexto, se generan interacciones, entre migrantes, población local, autoridades migratorias y policíacas en las que el sexo puede constituir el principal recurso para negociar la sobrevivencia y el tránsito hacia el lugar de destino, lo cual favorece las prácticas sexuales desprotegidas y el riesgo de infección por el VIH (Caballero, 2002).

En México y algunos países de Centroamérica, los esfuerzos para contrarrestar la epidemia se han enfocado a la prevención de la transmisión del $\mathrm{VIH}$ promoviendo políticas para el uso de sangre segura (Tapia, 2003) y la focalización de campañas que promueven el uso del condón (Rico, 1995) en poblaciones específicas como son los hombres que tienen sexo con hombres (HSH), trabajadoras sexuales (TS) y adolescentes.

Siendo la transmisión sexual la vía de transmisión del viH más frecuente (ONUSIDA, 2008) (CENSIDA, 2009) el condón, con una efectividad demostrada en la prevención de la transmisión (Davis, 1999) (Holmes, 2004) figura como un método apropiado. Sin embargo, en varios estudios se ha reportado un 
bajo uso del mismo (Salgado, 2000) (Salgado 1996) (Tapia 2004) (James 2005), una baja percepción de riesgo para infección de VIH en la población (Bronfman, 2002) (Herrera, 2002) e incluso se ha encontrado que la población considera la posibilidad de infectarse por el VIH como un evento aleatorio con posibilidad nula de modificarlo o fuera de su alcance (Infante, 2004).

Retomando la importancia de la prevención en el control del VIH/SIDA, el empleo del Modelo de Creencias en Salud (MCS) aplicado en la prevención del SIDA propone que para lograr un cambio en el comportamiento del individuo (adopción de medidas preventivas) se necesitan por lo menos tres elementos: la creencia o percepción de la importancia de un problema; la percepción de vulnerabilidad ante dicho problema; y la percepción de que tomar una medida para prevenirlo generará beneficios mayores que los inconvenientes derivados del esfuerzo para aplicarlos (Moreno, 2003) (Cabrera, 2001) (Soto, 1997). Así, consideramos que, previo a la adopción de medidas preventivas, se esperaría que el individuo identificará un beneficio que incentive dicha adopción. En el caso del condón, el beneficio esperado sería la protección contra las ITS y el viH para lo cual, la persona tendría primero que percibirse en riesgo ante éstas.

Si bien es cierto que existen otros beneficios que podrían ser tomados en cuenta en el análisis de este estudio, como son: uso de condón como método de planificación familiar y como objeto de erotización, identificamos a la prevención de ITS y VIH como el principal beneficio.

El objetivo de este trabajo es identificar los factores asociados a la disposición para uso de condón en la población residente de localidades con presencia de alto flujo migratorio, localizadas en Centroamérica y la frontera sur de México.

\section{MATERIAL Y MÉTODOS}

Para este estudio se emplearon los datos obtenidos de la aplicación de la «Encuesta a Población Local» del proyecto de investigación 'Poblaciones Móviles 
y VIH/SIDA en Centroamérica, México y Estados Unidos' llevado a cabo durante enero-mayo del 2001 por el Instituto Nacional de Salud Pública (INSP); Estos corresponden a los datos basales sobre percepciones, comportamientos y actitudes frente al VIH en la región. El estudio se ha mantenido diez años como parte del sistema de monitoreo de riesgos y salud de grupos móviles y migrantes.

Los comportamientos sexuales se encuentran vinculados a contextos sociales y culturales, los cuales no se modifican sustantivamente en periodos de corto plazo y requieren de intervenciones estructurales que incidan de largo plazo sobre las relaciones sociales comunitarias a nivel local. Por esta razón se decidió realizar un análisis secundario de los datos disponibles que sirva como punto de referencia para otros estudios sobre el tema.

Este estudio fue realizado en once estaciones de paso $^{1}$ ubicadas en ocho países: Belice, Costa Rica, El Salvador, Guatemala, Honduras, México, Nicaragua y Panamá. Estas estaciones de paso tienen en común la presencia de un alto flujo de población migrante, elevada densidad de bares, cantinas y trabajo sexual que involucra a personas de diferentes países de la región y a la misma población local. Así mismo, cuentan con la participación de Organizaciones de la Sociedad Civil (OSC) que trabajan en la prevención de VIH/SIDA o en la atención de migrantes.

En el diseño muestral del estudio original, para Belice, El Salvador, Guatemala, Honduras, México, Nicaragua y Panamá, se empleó el método de muestreo por conglomerados bietápico, con probabilidades proporcionales a los tamaños aproximados de los conglomerados. Costa Rica contaba con un marco muestral actualizado por lo que el muestreo utilizado fue aleatorio simple. El tamaño mínimo de la muestra se calculó, con un nivel de confianza del $95 \%$, un efecto de diseño de 2 y una tasa de no respuesta del 10\%. Las unidades de análisis son los integrantes de las viviendas de 16 años y más, y la encuesta se aplicó a una sola persona por vivienda, de preferencia al jefe(a) de familia.

${ }^{1}$ Con el término «estaciones de paso» se hace referencia a localidades fronterizas con una intensa movilidad poblacional y que son comunidades de tránsito para los grupos de poblaciones móviles hacia otro país (Bronfman et al., 2004). 
En todos los casos se muestreó buscando prevalencias de migración, información y conocimientos de VIH/SIDA, prevención de ITS y VIH, aceptación para uso de condón y actitudes de rechazo a poblaciones móviles. Para considerar las diferencias de diseño muestral, en el análisis estadístico de este estudio, se incluyó en el modelo multivariado como covariable a cada una de las once estaciones de paso en las que se levantó la encuesta dejándose como referencia a Costa Rica.

A los participantes del estudio se les proporcionó información sobre los objetivos del proyecto, se obtuvo el consentimiento informado y se les informó que estaban en la posibilidad de negarse a contestar alguna pregunta. El anonimato del informante se aseguró por medio de una codificación individual en la cual no se registró el nombre, el domicilio, ni las referencias particulares de los participantes. Este proyecto fue aprobado por los comités de Bioseguridad, Ética e Investigación del InSP.

Con la información recolectada en las once estaciones de paso se obtuvo un total de 4720 encuestas aplicadas en el estudio original, de las cuales, se eliminaron 643 observaciones (13.7\%) por no contar con datos de interés para algunas de las variables independientes (como el sexo del encuestado), y 274 (5.8\%) por no contar con datos de interés para la variable dependiente (no contestó la pregunta). En el análisis se incluyeron finalmente 3823 observaciones que sí cuentan con información de las variables seleccionadas para el estudio. Para el análisis de los datos se empleó el paquete estadístico Stata 9.0.

Para la creación del modelo estadístico multivariado propuesto en este estudio, se tomó de referencia el MCs para los cual se emplearon los siguientes elementos: 1) la percepción de riesgo para infección por ITS/VIH dentro de la dimensión de las percepciones individuales; 2) los factores sociodemográficos del individuo, el conocimiento que posee acerca de las ITS/VIH/SIDA y las actitudes ante el VIH/SIDA y uso de condón en la dimensión de factores modificantes; y 3) finalmente, la variable de interés «disposición para uso de condón» se corresponde con la dimensión de probabilidad de acción (Figura 1). 
FIGURA 1

Factores asociados a la disposición para uso de condón

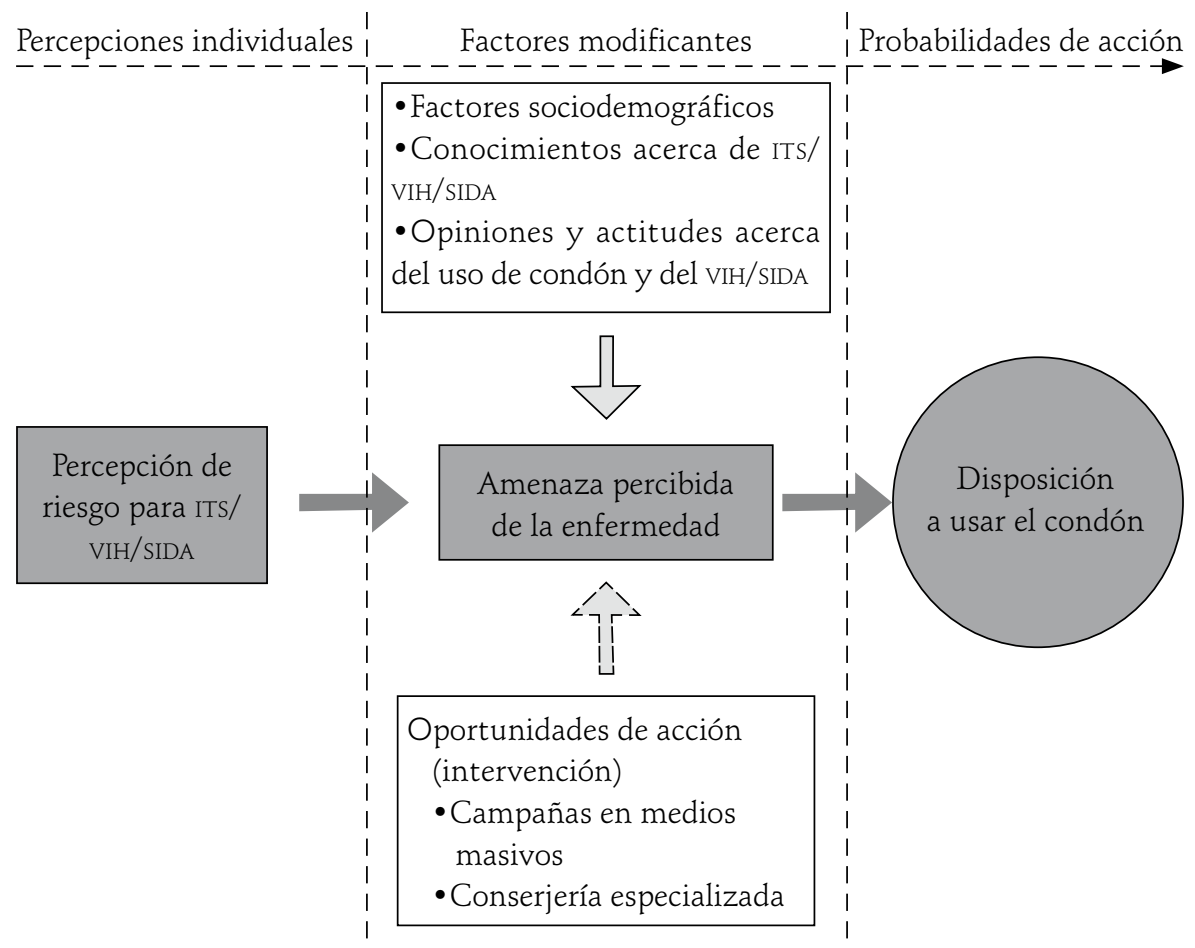

DESCRIPCIÓN DE LAS VARIABLES

Percepción de riesgo para infección por ITS/VIH. La percepción de riesgo se entiende como la apreciación del individuo de una situación particular que pone en peligro su persona (Del Pilar, 2002); y en este caso, como la apreciación subjetiva de su posibilidad para adquirir ITS y/o viH; Se exploró mediante la pregunta ¿Considera que la posibilidad de que usted se contagie con SIDA o alguna ITs es? y consta de cuatro categorías: Ninguna posibilidad, Baja, Media, Alta y No sabe/No contesta. 
Características sociodemográficas. En esta categoría se incluyen: «edad» (agrupada en 16-24, 25-44, 45-59 y 60 y más años de edad), «sexo» (hombre y mujer), «escolaridad» (sin escolaridad, 1-6, 7-9, 10-12, 13-15, 16-17 y 18-25 años), «Ocupación» (empleado en el hogar, estudiante y empleado fuera del hogar), «estado civil» (casados, solteros, viudos, divorciados y unión libre), y «lugar de residencia» (once estaciones de paso). En el modelo estadístico multivariado para hacer el comparativo del lugar de residencia del individuo se dejó de referencia a Costa Rica por ser el país con un IDH² mayor. (PNUD, 2006).

La variable «edad» se agrupó debido a que se esperaba encontrar una relación generacional en la disposición a usar condón, con mayor disponibilidad en el grupo de los más jóvenes. La variable «educación» se agrupó debido a que se encontraron efectos significativos al agruparla por grados académicos, efecto no observable si se analiza como variable continua.

Conocimientos acerca de las ITS y el VIH/SIDA. Mediante el empleo del análisis de correlación policórica (Kolenikov, 2006) se generó un índice de conocimientos de ITS/VIH/SIDA con tres categorías: «bajo», «medio», y «alto». Para la creación del índice se emplearon preguntas realizadas en la encuesta que exploran la información que tiene el encuestado acerca de la prevención y vías de transmisión del its y viH. (Tabla 1)

TABLA 1

Preguntas empleadas para la construcción del índice de conocimientos

1. ¿̇Ha oído hablar o sabe de las enfermedades o infecciones que se pueden contagiar al tener relaciones sexuales?

2. ¿Qué molestias puede tener una persona con alguna enfermedad de transmisión sexual?

3. ¿ iHa oído hablar del sidA?

4. $\quad$ iHay algo que pueda hacer una persona para evitar el contagio del SIDA?

5. ¿ ¿Me podría decir algunas formas en cómo se contagia el SIDA?

6. ¿ ¿Qué puede hacer una persona para evitar adquirir el SIDA?

Disposición a usar condón. La variable de interés disposición a usar condón (DUC) es una variable que se exploró mediante la pregunta: ว̇Usted usaría

2 El Índice de Desarrollo Humano (IDH) mide los progresos generales de un país en tres dimensiones básicas: educación, salud e ingreso económico. 
condón? Consta de tres opciones de respuesta: Sí, No, y No sabe/no contesta. La opción No Sabe/no contesta no se incluyó como una categoría de respuesta en la variable dependiente debido a que la información que proporciona no es de interés para los objetivos del estudio. Además no se encontró diferencia estadística entre las categorías No y No sabe/no contesta. Así, únicamente se emplearon para el análisis las opciones de respuesta: Sí y No, dejando fuera del análisis 274 datos (5.8\%) de la muestra original que corresponden a la opción No sabe/No contesta.

Plan de análisis

Para el análisis final se empleó un modelo multivariado de regresión logística; debido a que se ha demostrado que la presencia de una prevalencia alta (mayor del 20\%) de respuesta en la variable de interés, en un modelo de regresión logística, ocasiona que la razón de momios se sobrestime (Zhang, 1998) (Thompson, 1998) (Holcomb, 2001) (Schiaffino, 2003); se emplearon razones de prevalencia obtenidas mediante la fórmula de Zhang \& Yu e intervalos de confianza calculados mediante la fórmula de Miettinen ${ }^{3}$ para el ajuste final de los resultados que se presentan en el modelo multivariado.

Por la posibilidad teórica de la existencia de simultaneidad entre al variable independiente "percepción de riesgo» y la variable dependiente, se realizó el Test de Hausman para descartar endogeneidad (Prob F $=0.7264$ ) (Wooldridge, 2002) (Wooldridge, 2002), de la variable «percepción de riesgo» en el modelo final.

\section{RESULTADOS}

La población estudiada $(\mathrm{n}=3823)$ tienen una mediana para la edad de 36 años, con una distribución etárea concentrada (50\%) en el grupo de adultos jóvenes (de 25-44) años. La mediana de años de escolaridad de los encuesta-

${ }^{3}$ Referido por Schiaffino et al (2003). 
dos fue de 6 años (0-25 años), el 68\% refirió vivir con una pareja (casados/ unión libre), y el $82 \%$ tener hijos (Tabla 2 ).

TABLA 2

Características sociodemográficas de la población

\begin{tabular}{|c|c|c|c|c|c|c|}
\hline \multirow[b]{3}{*}{ VARIABLE } & \multirow[b]{3}{*}{ CATEGORÍAS } & \multicolumn{4}{|c|}{ SEXO } & \multirow{3}{*}{$\begin{array}{c}\text { TOTALPOR } \\
\text { CATEGORÍAS } \\
\mathrm{N}(\%)\end{array}$} \\
\hline & & \multicolumn{2}{|c|}{ MUJER } & \multicolumn{2}{|c|}{ HOMBRE } & \\
\hline & & $\mathrm{N}=2,550$ & $\%=66.7$ & $N=1,273$ & $\%=33.3$ & \\
\hline \multirow[t]{4}{*}{ Edad } & 16-24 años & 490 & 19.2 & 231 & 18.2 & $721(18.9)$ \\
\hline & 25-44 años & 1,375 & 54.0 & 555 & 43.6 & $1,930(50.5)$ \\
\hline & 45-59 años & 477 & 18.7 & 316 & 24.8 & $793(20.7)$ \\
\hline & 60 años y más & 208 & 8.1 & 171 & 13.4 & $379(9.9)$ \\
\hline \multirow{8}{*}{$\begin{array}{l}\text { Escolaridad } \\
\text { en años }\end{array}$} & & & & & & \\
\hline & Sin escolaridad & 291 & 11.4 & 110 & 8.7 & $401(10.5)$ \\
\hline & 1-6 años & 1,094 & 42.9 & 428 & 33.6 & $1,522(39.8)$ \\
\hline & 7-9 años & 457 & 17.9 & 273 & 21.5 & $730(19.1)$ \\
\hline & 10-12 años & 498 & 19.5 & 278 & 21.8 & $776(20.3)$ \\
\hline & 13-15 años & 125 & 4.9 & 89 & 7.0 & $214(5.6)$ \\
\hline & 16-17 años & 65 & 2.6 & 64 & 5.0 & $129(3.4)$ \\
\hline & $18-25$ años & 20 & 0.8 & 381 & 2.4 & $51(1.3)$ \\
\hline \multirow[t]{4}{*}{ Ocupación } & Empleado en el hogar & 1,594 & 62.5 & 86 & 6.8 & $1,680(43.9)$ \\
\hline & Estudiante & 98 & 3.9 & 80 & 6.2 & $178(4.7)$ \\
\hline & Empleado fuera del hogar & 847 & 33.2 & 1,089 & 85.6 & $1,936(50.6)$ \\
\hline & Sin información & 11 & 0.4 & 128 & 1.4 & $29(0.8)$ \\
\hline \multicolumn{7}{|l|}{ Estado } \\
\hline \multirow[t]{7}{*}{ civil } & Casado/a & 1,028 & 40.3 & 584 & 45.9 & $1,612(42.2)$ \\
\hline & Divorciado/a & 89 & 3.5 & 29 & 2.3 & $118(3.1)$ \\
\hline & Unión libre & 703 & 27.6 & 300 & 23.6 & $1,003(26.2)$ \\
\hline & Viudo/a & 156 & 6.1 & 35 & 2.7 & $191(5.0)$ \\
\hline & Soltero/a & 534 & 20.9 & 315 & 24.7 & $849(22.2)$ \\
\hline & Otros & 36 & 1.4 & 6 & 0.5 & $42(1.1)$ \\
\hline & Sin información & 4 & 0.2 & 4 & 0.3 & $8(0.2)$ \\
\hline \multirow[t]{3}{*}{ Hijos } & Sí & 2,190 & 85.9 & 934 & 73.4 & $3,124(81.7)$ \\
\hline & No & 359 & 14.1 & 334 & 26.2 & $693(18.1)$ \\
\hline & Sin información & 1 & 0.0 & 5 & 0.4 & $6(0.2)$ \\
\hline Total & & 2,550 & 100 & 1,273 & 100 & $3,823(100)$ \\
\hline
\end{tabular}

Fuente: Elaboración propia con datos de la Encuesta Poblacional sobre ITS/VIH/SIDA y migración del proyecto «Poblaciones Móviles y VIH/Sida en Centroamérica, México y Estados Unidos». 
TABLA 3

Conocimientos acerca del viH/SIDA

\begin{tabular}{|c|c|c|c|c|c|c|}
\hline \multirow[b]{3}{*}{ VARIABLE } & \multirow[b]{3}{*}{ CATEGORÍAS } & \multicolumn{4}{|c|}{ SEXO } & \multirow{3}{*}{$\begin{array}{l}\text { TOTAL POR } \\
\text { CATEGORÍAS } \\
\mathrm{N}(\%)\end{array}$} \\
\hline & & \multicolumn{2}{|c|}{ MUJER } & \multicolumn{2}{|c|}{ HOMBRE } & \\
\hline & & $\mathrm{N}=2,550$ & $\%=66.7$ & $\mathrm{~N}=1,273$ & $\%=33.3$ & \\
\hline \multirow{3}{*}{$\begin{array}{l}\text { ¿Ha oído hablar o sabe de las enferme- } \\
\text { dades o infecciones que se pueden con- } \\
\text { tagiar al tener relaciones sexuales? }\end{array}$} & Sí & 2,449 & 96.0 & 1,222 & 96.0 & $3,671(96.0)$ \\
\hline & No & 87 & 3.4 & 49 & 3.8 & $136(3.6)$ \\
\hline & Sin información & 14 & 0.6 & 2 & 0.2 & $16(0.4)$ \\
\hline \multirow{8}{*}{$\begin{array}{l}\text { ¿ué molestias puede tener una per- } \\
\text { sona con alguna enfermedad de trans- } \\
\text { misión sexual? }\end{array}$} & Flujo/secreción genital & 424 & 16.6 & 273 & 21.5 & $697(18.2)$ \\
\hline & Comezón/ardor genital & 147 & 5.8 & 105 & 8.2 & $252(6.6)$ \\
\hline & Ámpulas/hinchazón genital & 58 & 2.3 & 26 & 2.0 & $84(2.2)$ \\
\hline & Dolor al orinar & 176 & 6.9 & 119 & 9.3 & $295(7.7)$ \\
\hline & Más dos síntomas & 186 & 7.3 & 109 & 8.6 & $295(7.7)$ \\
\hline & Otros & 411 & 16.1 & 188 & 14.8 & $599(15.7)$ \\
\hline & No aplica & 101 & 4.0 & 51 & 4.0 & $152(4.0)$ \\
\hline & No sabe/no contesta & 1,047 & 41.0 & 402 & 31.6 & $1,449(37.9)$ \\
\hline \multirow{3}{*}{$\begin{array}{l}\text { ¿Hay algo que pueda hacer una perso- } \\
\text { na para evitar contagiarse con una en- } \\
\text { fermedad o infección de transmisión } \\
\text { sexual? }\end{array}$} & Sí & 2,262 & 88.7 & 1,165 & 91.5 & $3,427(89.6)$ \\
\hline & No & 71 & 2.7 & 25 & 1.9 & $96(2.5)$ \\
\hline & Sin Información & 217 & 8.5 & 83 & 6.5 & $300(7.8)$ \\
\hline \multirow{6}{*}{$\begin{array}{l}\text { ¿Cómo se puede evitar el contagio de } \\
\text { una enfermedad o infección de trans- } \\
\text { misión sexual? }\end{array}$} & Usar condón & 1,521 & 59.7 & 848 & 66.7 & $2,369(62.0)$ \\
\hline & Abstinencia sexual & 365 & 14.4 & 147 & 11.6 & $512(13.4)$ \\
\hline & Fidelidad/monogamia & 288 & 11.3 & 117 & 9.2 & $405(10.6)$ \\
\hline & Otro & 71 & 2.7 & 39 & 3.0 & $110(2.9)$ \\
\hline & No aplica & 288 & 11.2 & 108 & 8.4 & $396(10.3)$ \\
\hline & No sabe/no contesta & 17 & 0.7 & 14 & 1.1 & $31(0.8)$ \\
\hline \multirow[t]{3}{*}{ ¿Ha oído hablar del SIDA? } & Sí & 2,515 & 98.6 & 1,256 & 98.7 & $3.771(98.6)$ \\
\hline & No & 20 & 0.8 & 12 & 0.9 & $32(0.9)$ \\
\hline & Sin información & 15 & 0.6 & 5 & 0.4 & $20(0.5)$ \\
\hline \multirow{3}{*}{$\begin{array}{l}\text { ¿Hay algo que pueda hacer una persona } \\
\text { para evitar el contagio del SIDA? }\end{array}$} & Sí & 2,320 & 91.0 & 1,192 & 93.7 & $3,512(91.9)$ \\
\hline & No & 68 & 2.7 & 22 & 1.7 & $90(2.3)$ \\
\hline & Sin información & 162 & 6.3 & 59 & 4.6 & $221(5.8)$ \\
\hline \multirow{6}{*}{$\begin{array}{l}\text { ¿Me podría decir algunas formas en } \\
\text { cómo se contagia el SIDA? }\end{array}$} & Contacto sexual & 2,131 & 83.5 & 1,079 & 84.7 & $3,210(83.9)$ \\
\hline & Contacto con sangre contaminada & 112 & 4.3 & 42 & 3.3 & $154(4.0)$ \\
\hline & Madre infectada contagia a bebé & 111 & 4.3 & 58 & 4.5 & $169(4.4)$ \\
\hline & Lactancia & 0 & 0.0 & 2 & 0.1 & $2(0.0)$ \\
\hline & Otro & 32 & 1.2 & 9 & 0.7 & $41(1.0)$ \\
\hline & No sabe/no contesta & 164 & 6.4 & 83 & 6.5 & $247(6.4)$ \\
\hline \multirow{8}{*}{$\begin{array}{l}\text { ¿Qué puede hacer una persona para } \\
\text { evitar adquirir el SIDA? }\end{array}$} & Usar Condón & 1,362 & 53.4 & 774 & 60.8 & $2,136(55.9)$ \\
\hline & Abstinencia sexual & 465 & 18.3 & 193 & 15.2 & $658(17.2)$ \\
\hline & Fidelidad & 170 & 6.7 & 76 & 6.0 & $246(6.5)$ \\
\hline & No reutilizar agujas & 103 & 4.0 & 49 & 3.8 & $152(4.0)$ \\
\hline & Usar sangre segura & 34 & 1.3 & 17 & 1.3 & $51(1.3)$ \\
\hline & Otros & 125 & 4.9 & 52 & 4.1 & $177(4.6)$ \\
\hline & No sabe/no contesta & 291 & 11.4 & 112 & 8.8 & $403(10.5)$ \\
\hline & Total & 2,550 & 100 & 1,273 & 100 & $3,823(100)$ \\
\hline
\end{tabular}

Fuente: Elaboración propia con datos de la Encuesta Poblacional sobre ITS/VIH/SIDA y migración del proyecto «Poblaciones Móviles y VIH/SIDA en Centroamérica, México y Estados Unidos». 
La población tiene información sobre ITS y VIH/SIDA; sabe que las ITS y el VIH se transmiten por vía sexual, que son prevenibles e identifican el uso de condón como método preventivo (Tabla 3). El 100\% refirió saber para qué sirve el condón y el $66 \%$ lo identificó como método preventivo de ITs. En relación con la información sobre sexualidad el 77\% refirió que durante su infancia sus padres no les habían hablado de temas relacionados con la sexualidad. 88\% respondió, que sí se debe de hablar de sexo con los menores de 15 años de edad y 97\% estaría de acuerdo en que en la escuela les dieran información sobre ITS y VIH/SIDA a sus hijos y hermanos menores.

Al explorar la percepción de riesgo del individuo para contraer una ITS o $\mathrm{VIH}$, se encontró que $45 \%$ de los encuestados no se percibe en riesgo, $22 \%$ considera que su riesgo es bajo, $12 \%$ que es regular, $9 \%$ que es alto y $11 \%$ no respondió a la pregunta. Respecto a la disposición para usar condón, $65 \%$ contestó que sí usaría el condón y 35\% que no. Los hombres refieren estar más dispuestos a usar condón que las mujeres (76 y 60\%) ( $p<0.001)$.

\section{Modelo logístico multivariado}

Se realizó un modelo multivariado de regresión logística. Las variables independientes incluidas en el análisis se pueden agrupar en cuatro categorías: factores sociodemográficos, conocimientos acerca de ITS/VIH/SIDA, actitudes ante el VIH/siDA y uso de condón, y percepción de riesgo (Tabla 4).

La razón de prevalencias (RP) muestra que los hombres están más dispuesto a usar condón que las mujeres (1.23) Al incrementar la edad del individuo disminuye la disposición a usar condón; la diferencia entre grupos de edad fue estadísticamente significativa ( $p<0.05)$. Respecto al estado civil, se observó que los casados, los divorciados y los viudos tienen menor disposición a usar condón que los solteros.

Se realizó una interacción de sexo con estado civil y se encontró que en el grupo de hombres, los casados (RP 0.77), los hombres viudos (RP 0.61) y en unión libre (RP 0.77) tienen menor disposición a usar condón que los solteros $(p<0.05)$, esto no fue significativo en el grupo de mujeres. 
Los individuos con escolaridad mayor de seis años tienen una mayor disposición para usar condón que quienes tienen menos años de escolaridad. Se efectuó una interacción de sexo con escolaridad encontrándose que en el grupo de mujeres quienes tienen escolaridad de más de seis años muestran mayor disposición a usar condón. Es decir el incrementar la escolaridad en las mujeres tendría un efecto positivo en la disposición a usar condón, efecto no observado en los hombres.

La percepción de riesgo para infección por ITS/VIH/SIDA está asociada a la disposición para usar condón; quienes se perciben en riesgo bajo (RP1.16), medio (RP1.20) y alto (RP1.21) muestran más disposición a usar condón que quienes se perciben sin ningún riesgo.

\section{DISCUSIÓN}

En este estudio, la población que está dispuesta a usar condón se compone por: hombres, población joven y soltera, estudiantes, con escolaridad mayor de seis años, con nivel medio-alto de conocimientos de ITS y VIH/SIDA, que recomiendan a todas las personas sexualmente activas usar condón, que se muestran interesados en recibir información acerca de ITS y VIH/SIDA, consideran que se debe dar más información del tema a los jóvenes y que se perciben en riesgo para contraer ITS y/o VIH.

La disposición a usar condón se percibe como una actitud positiva ante la medida preventiva y se considera como un paso previo para el uso de condón (Herrera 2002). Si bien el estar dispuesto a usar condón no garantiza el uso del mismo, el rechazo o la no disposición a usarlo está asociado a prácticas sexuales desprotegidas. La población estudiada reconoce ampliamente que las ITS y el VIH son prevenibles e identifica al condón como un método efectivo de prevención por lo que se esperaría que estuvieran dispuestos a usarlo. Sin embargo, 35\% de la población encuestada refirió no estar dispuesta a usarlo.

La mayor disposición de los hombres para usar condón puede explicarse porque el condón es un dispositivo que utiliza el hombre y que requiere de poca 


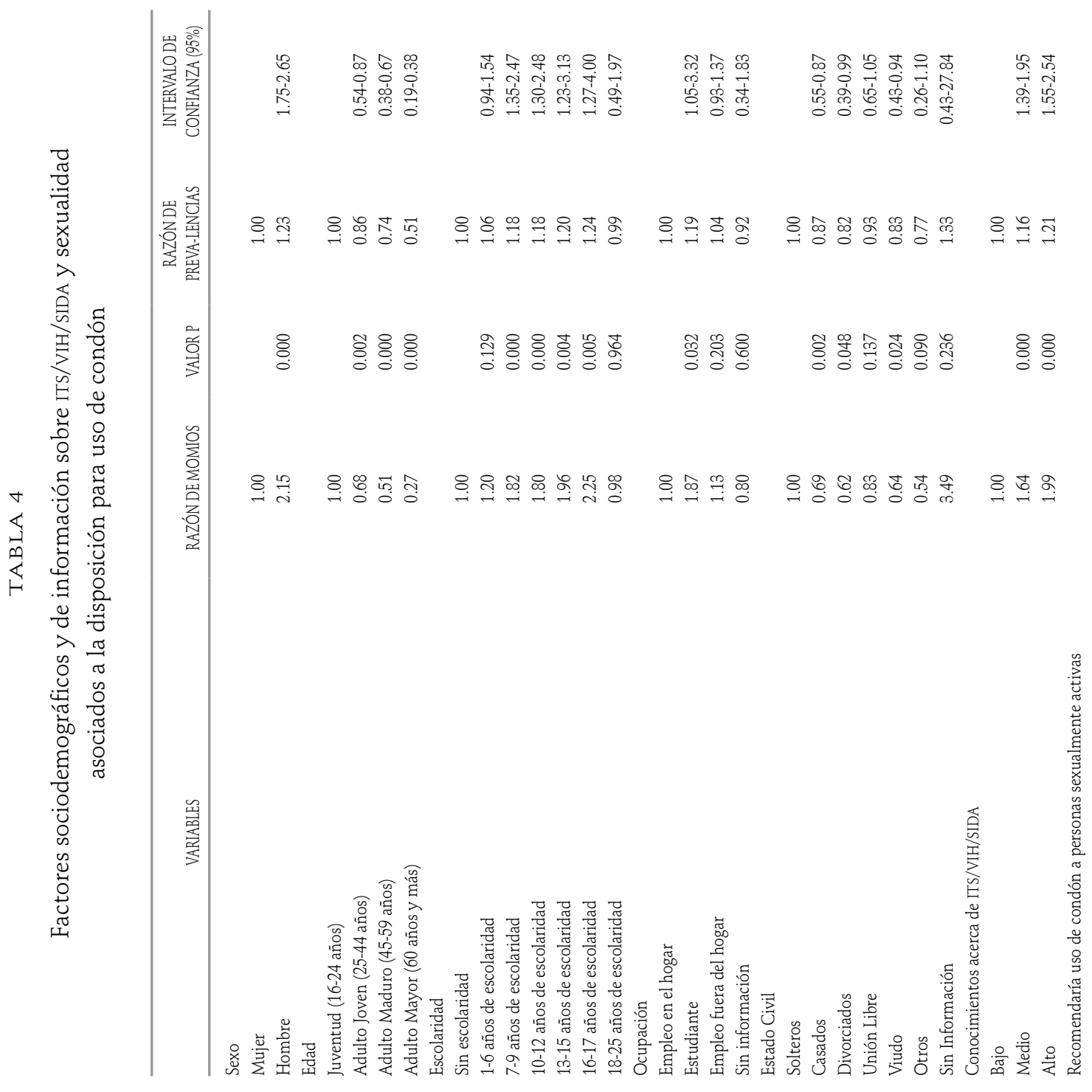




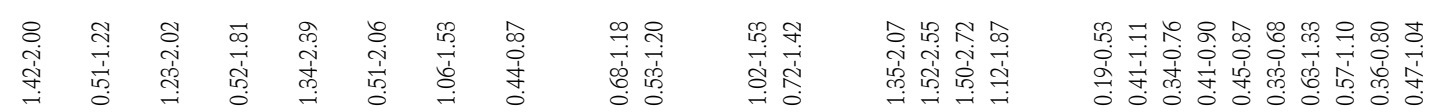

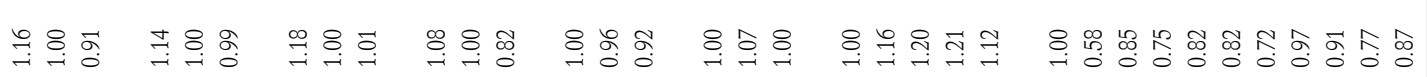

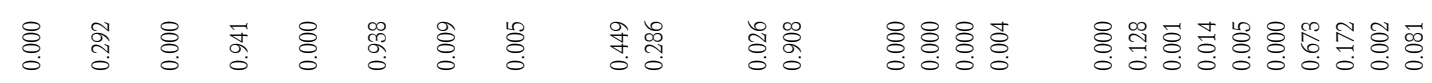

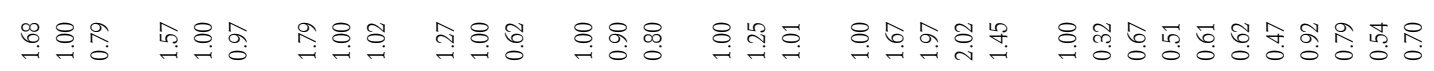

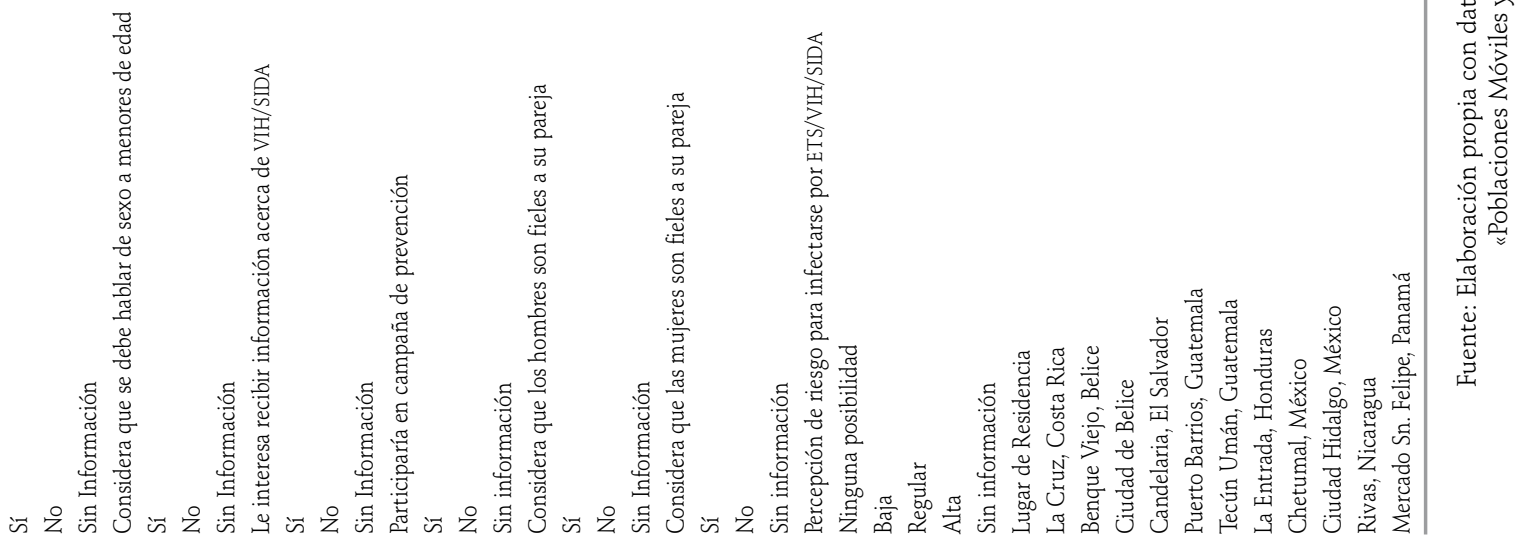


participación por parte de la mujer. Lo cual se refuerza por el escaso poder de negociación de la mujer para el uso de condón determinada por los roles de género de las sociedades latinoamericanas, en las cuales, se espera que la mujer no tenga experiencia sexual previa al matrimonio y que sea el hombre el experimentado (Amuchástegui, 1996) (Szasz, 1998).

Se observa una tendencia generacional en la disposición a usar condón, es así como, los más jóvenes están más dispuestos a usarlo. Esto probablemente represente un resultado de los esfuerzos de los programas de salud en la implementación de campañas preventivas focalizadas a grupos específicos de población como los jóvenes. El hecho de que sea el grupo de jóvenes el que muestra mayor disposición a usar condón resulta positivo para la prevención. Sin embargo, los jóvenes y especialmente las mujeres, frecuentemente tienen prácticas sexuales con personas de mayor edad (Gutiérrez, 2006), las cuales tienen menor disposición a usar condón. Por lo anterior, es necesario fortalecer las intervenciones existentes con especial énfasis en la perspectiva de género ya que las relaciones inequitativas es un factor que incrementa la vulnerabilidad al riesgo.

La escolaridad del individuo es un factor relacionado con las condiciones del contexto en que se desarrolla y puede modificar la percepción que tiene de la enfermedad como amenaza; es una variable en la que se puede incidir a través de acciones programáticas de educación. Además, mediante el incremento de la escolaridad se puede facilitar el empoderamiento de la mujer aumentando su capital social y con ello su capacidad de negociación del condón como método de prevención de ITS y VIH.

Respecto a la percepción de riesgo para ITS y VIH como determinante para la adopción de medidas preventivas, comprobamos la importancia de la percepción de riesgo para ITS y VIH como predictor de la disposición a usar el condón planteada en el modelo (Figura 1). No obstante, aproximadamente la mitad de la población encuestada no se percibe en riesgo para infectarse con alguna ITS, aún siendo habitantes de países con tasas altas de prevalencia de ITS y VIH/SIDA de acuerdo con el Informe de ONUSIDA del 2008. Lo anterior puede estar relacionado con la concepción de que el VIH/SIDA es un problema específico de algunos grupos de la población (Infante 2004). 
Los resultados de este estudio son consistentes con los presentados en otros países (ONUSIDA, 2004) (Gayet, 2003) (Prata, 2005) (Bobrova, 2005) (Gayet, 2007) en los que se ha documentado que el incremento de la escolaridad tiene un efecto positivo en la prevención de VIH/sIDA; Así mismo se ha documentado que son los hombres, solteros, jóvenes, estudiantes y empleados fuera del hogar quienes usan más el condón.

Consideramos conveniente la realización de encuestas nacionales sobre sexualidad, las cuales nos permitirán caracterizar mejor a las poblaciones conociendo su identidad sexual, prácticas sexuales, nivel de información sobre ITS/VIH, percepción de riesgo para adquirirlas; así cómo, las prácticas preventivas empleadas para evitarlas. Lo anterior, será de gran utilidad para el planteamiento de estrategias preventivas específicas y sensibles a las necesidades de los distintos grupos poblacionales.

La principal limitación de este estudio es que la información recabada al explorar la variable dependiente, no permite discriminar la disposición a usar en condón en situaciones diferenciadas, como por ejemplo, el tipo de pareja o compañero sexual (parejas estables y parejas ocasionales), en donde se ha documentado que hay un uso diferenciado del condón. Tampoco tenemos elementos que nos permitan inferir que la percepción de riesgo para ITS/VIH referida por los encuestados está directamente relacionada con sus prácticas sexuales.

\section{BIBLIOGRAFÍA}

Amuchástegui-Herrera, A. (1996), «El significado de la virginidad y la iniciación sexual. Un relato de investigación», en I. Szasz y S. Lerner, Para comprender la subjetividad, Investigación cualitativa en salud reproductiva y sexualidad, México D.F., El Colegio de México, pp. 137-172.

Bobrova, Natalia, Oleg Sergeev, Tatyana Grechukhina, Saidi Kapiga (2005), «SocialCongnitive predictors of consistent condom use among people in Moscow», Perspectives on Sexual and Reproductive Health, vol. 37, núm. 4, pp. 174-178.

Bronfman, Mario, René Leyva, Mirka Negroni, Marta Caballero, César Infante y Silvia Magali et al. (2003), «Migración género y SIDA: contextos de vulnerabilidad», Género y Salud en Cifras, México D.F., vol. 1, pp. 8-12. 
René Leyva, Mirka Negroni y C. Herrera (2003), «El siDA en las fronteras», en D. Alarcón, S. Ponce de León, El sIDA en México veinte años de la epidemia, México D.F., El Colegio Nacional, pp. 169-201.

, René Leyva, Mirka Negroni y Celina Rueda (2002), Mobile populations and HIV/AIDS in Central America and Mexico: research for action, AIDS, vol. 16, S42-S49.

Caballero, Marta, Anahi Dreser, René Leyva, Celina Rueda y Mario Bronfman (2002), Migration, gender and HIV/AIDS in Central America and Mexico, XIV International Aids Conference, Barcelona, Monduzzi Edit., pp. 263-267.

Cabrera, G., J. Tascón, D. Lucumí (2001), «Creencias en Salud: historias, constructos y aportes al modelo», Rev. Fac. Nac. Salud Pública, vol. 19, pp. 91-101.

Centro Nacional para la Prevención y el Control del vih/sida, México (Censida), Casos de sida en México. Casos acumulados por categoría de transmisión y sexo. Cifras al 31 de marzo de 2009. Consultado el 25 de octubre de 2009 en http://www.censida.salud.gob.mx/interior/cifras.html

DAVIs, Karen R. y Susan C. Weller (1999), "The effectiveness of condoms in reducing heterosexual transmission of HIV», Family Planning Perspectives, vol. 31, pp. 272-279.

Del Pilar, María (2002), El riesgo en salud: entre la visión del lego y el experto, Bogotá, Unibiblos, pp. 1-75.

GAYET, Cecilia, Fátima Juárez, Laura Pedrosa y Carlos Magis (2003), «Uso del condón entre adolescentes mexicanos para la prevención de las infecciones de transmisión sexual», Salud Pública de México, vol. 45: S632-S640.

y Patricio Solis (2007), «Sexualidad saludable de los adolescentes: la necesidad de políticas basadas en evidencias», Salud Pública de México, edición especial XII Congreso de Investigación en Salud Pública, E47-E51.

Gutiérrez, J.P., S.M. Bertozzi, C. Conde-Gonzalez y M.A. Sanchez-Aleman (2006) "Risk behaviors of 15-21 years old in Mexico lead to high prevalence of sexually transmitted infections: results of a survey in disadvantaged urban areas», BMC Public Health, vol. 6, pp. 1-11.

Herrera, Cristina y Lourdes Campero (2002), «La vulnerabilidad e invisibilidad de las mujeres ante el VIH/SIDA: constantes y cambios en el tema», Salud Pública de México, vol. 44, pp. 554-64.

HolcomB, W.L., T. Chaiworapongsa, D.A. Luke y K.D. Burgdorf (2001), «An Odd measure of risk: use and misuse of the Odds Ratio», Obstetrics \& Gynecology, vol. 98, pp. 685-688.

Holmes, K.K., R. Levine, M. Weaver (2004), «Effectiveness of condoms in preventing sexually transmitted infections", Bulletin of the World Health Organization, vol. 82, pp. $454-461$. 
Infante Xibille, César, René Leyva Flores, Marta Caballero García, Claudia Guerrero, Magali Cuadra Silvia y Mario Bronfman (2004), «VIH/SIDA y rechazo a migrantes en contextos fronterizos», Migración y desarrollo, pp. 45-53.

James, Essein, E. Ross, W. Michael, M. Fernandez-Esquer, L. Williams Mark (2005) «Reported condom use and condom use difficulties in street outreach samples of men of four racial and ethnic backgrounds", International Journal of std AIDS, vol. 16, pp. 739-743.

Joint United Nations Programme on hiv/Aids unaids, International Organization for Migration IOM (1998), "Migration and AIDS», International Migration, vol. 36(4), pp. 445-68.

Kolenikov, S., G. Angeles (2006), The Use of Discrete Data in pca: Theory, Simulations and Applications to Socioeconomic Indices. Consultado el 15 de agosto de 2009 en https://www.cpc.unc.edu/measure/publications/pdf/wp-04-85.pdf

Moreno San Pedro, Emilio, Jesús Gil Roales-Nieto (2003), «El Modelo de Creencias en Salud: revisión teórica, consideración crítica y propuesta alternativa. I: Hacia un análisis funcional de las creencias en salud», International Journal of Psychology and Psychological Therapy, vol. 3, pp. 91-109, España.

ONUSIDA, Infecciones por VIH-epidemiología, en ONUSIDA, Situación de la epidemia de SIDA 2004. Consultado el 25 mayo de 2005 en http://www.unaids.org , Informe sobre la epidemia mundial de SIDA 2008. Consultado el 25 de septiembre de 2009 en http://www.unaids.org/es/KnowledgeCentre/HIvData/ GlobalReport/2008/2008_Global_report.asp

Prata N., F. Vahidnia, A. Fraser (2005), "Gender and relationship differences in condom use among 15-24 years old in Angola», International Family Planning Perspectives, vol. 31, pp. 192-199.

Programa de las Naciones Unidas para el Desarrollo (pnud) (2009). Informe sobre desarrollo humano 2009. Superando barreras: movilidad y desarrollo humanos. La cooperación internacional ante una encrucijada, Grupo Mundi-Prensa, pp. 228-233.

Rico, B., M. Bronfman y C. del Río-Chiriboga (1995), "Las campañas contra el SiDA en México: ว̇Los sonidos del silencio o puentes sobre aguas turbulentas?», Salud Pública de México, vol. 37, pp. 643-653.

Salgado de Snyder, V. Nelly, Andrea Acevedo, María de Jesús Díaz-Pérez y Alicia Saldívar-Garduño (2000), "Understanding the sexuality of mexican-born women and their risk for HIV/AIDS», Psychology Women Quarterly, vol. 24, pp. 100-109.

, María de Jesús Díaz-Pérez y M Maldonado (1996), «AIDs: Risk behaviors among rural mexican women married to migrant workers in the united status», AIDS Education and Prevention, Nueva York, vol. 8, pp. 134-142. 
Schiaffino, Anna, M. Rodríguez, M. Pasarín, E. Regidor, C. Borrellb y E. Fernández (2003), «วंOdds ratio o razón de proporciones? Su utilización en estudios transversales», Gaceta Sanitaria, Barcelona, España, vol. 17, pp. 70-74.

Shedlin, M.G., E. Drucker, C.U. Decena, S. Hoffman, G. Bhattacharya, S. Beckford, R. Barreras (2006), "Immigration and HIV/AIDs in the New York Metropolitan Area», Journal of Urban Health, El Paso, Texas, vol. 83(1), pp. 43-58.

, C.U. Decena y D. Oliver-Velez (2007), «Initial acculturation and HIV risk among new Hispanic immigrants», Journal of the National Medical Association, vol. $97(7$ Suppl): 32S-37S.

Soto Mas, F., J. Lacoste Marín, R. Papenfuss y A. Gutiérrez León (1997), «El Modelo de Creencias en Salud. Un enfoque teórico para la prevención del SIDA», Revista Española de Salud Pública, España, vol. 71: 335-341.

SzASz, Ivonne (1998), "Primeros acercamientos al estudio de las dimensiones sociales y culturales de la sexualidad en México», en I. Szasz, S. Lerner, Sexualidades en México. Algunas aproximaciones desde la perspectiva de las Ciencias Sociales, México D.F., El Colegio de México, pp. 11-31.

TAPIA, R., E. Bravo y P. Uribe (2003), «Evolución de la epidemia de sIDA en México», en D. Alarcón, S. Ponce de León, El SIDA en México veinte años de la epidemia, México D.F., El Colegio Nacional, pp. 19-48.

TApiA, V., E. Arillo, B. Allen, A. Angeles, A. Cruz y E. Lazacano (2004), «Associations among condom use, sexual behavior, and knowledge about HIV/AIDS. A study of 13,293 public school students», Archives of Medical Research, vol. 35, pp. 334-343.

Thompson, M.L., J.E. Myers y D. Kriebel (1998), «Prevalence odds ratio or prevalence ratio in the analysis of cross sectional data: what is to be done?», Occup Environ Med, vol. 55, pp. 272-277.

WoOlDRIDGE, J. (2002), "Estimación con variables instrumentales y mínimos cuadrados bietapicos», en Wooldridge, Introducción a la econometría, un enfoque moderno, México D.F., Thomson Learning, pp. 461-500.

(2002), «Test for endogeneity», en J. Wooldrige, Econometric Analysis of Cross Section and Panel Data, Massachusetts, The mit Press, pp. 118-122.

ZHANG, Jun y F. Yu Kai (1998) «What's the Relative Risk? A method of correcting the odds ratio in cohort studies of common outcomes», JAMA, vol. 280, pp. 1690-1691. 